\title{
ACCIONES INTERINSTITUCIONALES DE VINCULACIÓN Y TRANSFERENCIA EN EL SECTOR APÍCOLA Y EN LA COMUNIDAD DE CHACO Y CORRIENTES
}

\section{Interinstitutional linkage and transference in the beekeeping sector and communities fron Chaco and Corrientes}

\author{
Salgado Laurenti, Cristina R. ${ }^{1-2}$; Sobrado, Sandra V. ${ }^{2-3}$; Ruiz Díaz, Juan D. ${ }^{3-4}$; Rusas, \\ Victor $^{5}$ \\ ${ }^{1-}$ FCA. UNNE. Sgto. Cabral 2131. CP 3400. Corrientes. Argentina. e-mail: crsalgado2009@gmail.com \\ ${ }^{2-I B O N E}$ (UNNE-CONICET). Sgto. Cabral 2131. CP 3400. Corrientes. Argentina. \\ ${ }^{3-F a C E N A . ~ U N N E . ~ A v . ~ L i b e r t a d ~ 5470 . ~ C P ~ 3400 . ~ C o r r i e n t e s . ~ A r g e n t i n a . ~ e-m a i l: ~ s o b r a d o s a n d r a @ g m a i l . c o m ~}$ \\ ${ }^{4-L A B A P I}$. Av. Libertad 5470. CP 3400. Corrientes. Argentina. e-mail: jdrdiaz@yahoo.com \\ ${ }^{5-I N T A . ~ M a r c o s ~ B r i o l i n i ~ 750, ~ E E A ~} 3505$ Colonia Benítez, Chaco. Argentina. CP 3505. Chaco. Argentina. \\ e-mail: rusas.victor@inta.gob.ar
}

\section{RESUMEN}

Este trabajo tiene como objetivo mostrar una serie de acciones de vinculación y trasferencia realizadas por un grupo interdisciplinario de profesionales. Estos pertenecen a FaCENA y FCA (UNNE), CONICET, INTA, e INTI. Incluye también una componente docente que busca formar, en tareas extensionistas, a los estudiantes de grado involucrados en los Proyectos del Programa "La Universidad en el Medio". Tiene como finalidad dar a conocer la calidad de las mieles chaqueñas, principalmente las producidas en el Sitio Ramsar y localidades cercanas, asociada al origen floral para generar estrategias de implementación de valor agregado. Con el abordaje de diferentes problemáticas en la producción de miel, las acciones se realizaron con un grupo apicultores del Chaco, que pertenecen a dos cooperativas (COPAP [Margarita Belén] y Los Palmares [Basail]) y a una asociación apícola (Asociación de Productores Apícolas [Charadai]). Esta última no está incluida dentro del Sitio Ramsar. Todas las intervenciones apostaron al fortalecimiento del sector apícola de la región, mejorar la producción y aumentar el consumo de miel principalmente. Durante las jornadas de la "Semana de Miel", se difundió sobre las propiedades naturales de los productos apícolas, además de fomentar el consumo de los productos primarios de las abejas y sus derivados. Se realizaron acciones con niños del nivel inicial promoviendo el consumo de miel, el cuidado y protección de las abejas como agentes polinizadores y conservadores de la biodiversidad.

Palabras clave: apicultor es - miel - origen - calidad - Nordeste.

\begin{abstract}
The aim of this work is to show a set of actions of correlation and transfer carried out by an interdisciplinary group of professionals. They belong to FaCENA and FCA (UNNE), CONICET, INTA, and INTI. It also includes a teaching component that attempts to train, in extensionist tasks, the undergraduate students involved in the Projects of the Program "La Universidad en el Medio". It looks to raise the quality of different types of honeys from Chaco, mainly those produced in Ramsar Site and nearby villages, associated with the floral origin to generate value-added implementation strategies. In order to approach different problems in honey production, the actions were carried out with a group of beekeepers from Chaco, who belong to two cooperatives (COPAP [Margarita Belén] and "Los Palmares" [Basail]) and a beekeeping association (Asociación de Productores Apícolas [Charadai]). The latter is not included within the Ramsar Site. All the interventions were focused on strengthening the region's beekeeping sector, improving production and increasing the consumption of honey in particular. During the events "Semana de la Miel", the natural properties of beekeeping products were made public, as well as promoting the consumption of primary bee products and their derivatives. The actions were performed with children of the initial level promoting the consumption of honey, the care and protection of the bees as pollinating agents and biodiversity keepers.
\end{abstract}

Key words: beekeepers - honey - origin - quality - Northeast.

Recibido: 02/jun/2020. Aceptado: 08/jul/2020 


\section{INTRODUCCIÓN}

La Universidad asume un compromiso social al responder demandas concretas del entorno que la rodea. En este sentido, se organiza en un sistema de integración sistémica que incluye: docencia, investigación y extensión como procesos claves. Cuando la institución académica se involucra en su conjunto para satisfacer las demandas sociales, la extensión es un factor clave para obtener el correspondiente crecimiento cultural de la universidad. La extensión garantiza un vínculo social más amplio y dinámico, que propicia la identificación, comunicación y actividad conjunta de los universitarios y la población en general (González González y Fernández Larrea 2019).

El sector agropecuario constituido por pequeños productores requiere especial atención por parte de todas las instituciones estatales: las académicas como la Universidad, las gubernamentales como lo son los Ministerios y las de transferencia tecnológica como el INTA.

La apicultura es una actividad productiva amigable con el ambiente, puesto que se puede realizar en ambientes naturales o en agroecosistemas, es inclusiva ya que puede ser practicada por personas de distintas edades y escala socio-cultural. El apicultor se define como la persona que se dedica a la cría de las abejas y al aprovechamiento de los productos, primarios y secundarios, que ellas elaboran.

En la región del Nordeste Argentino la apicultura es una actividad económica en crecimiento y constituida por pequeños productores agrupados en cooperativas o asociaciones, las cuatro provincias que integran esta región presentan diferente grado de desarrollo y organización. En este sentido, la provincia del Chaco es la más organizada y con mayor estructura productiva. En la mencionada provincia, la actividad apícola cuenta con una importante diversidad de ambientes naturales a partir de los cuales se producen distintos tipos de mieles de acuerdo a su origen floral (Salgado 2006a, Salgado et al. 2014). Estas mieles, producidas principalmente a base de néctar de especies del monte nativo (Salgado et al. 2017), poseen valiosas propiedades fisicoquímicas (Salgado y Maidana 2014) que permiten diferenciarlas de mieles de pradera producidas en la región pampeana y en otras partes de nuestro país. Algunos ejemplos pueden ser miel de quebracho colorado (Schinopsis balansae Engl.) y miel de algarrobo (Prosopis alba Griseb.). Por su parte, la provincia de Corrientes produce principalmente mieles de Eucalyptus y Citrus (Salgado y Pire 1998, 1999), con notables propiedades fisicoquímicas (Bertona et al. 2014, Fechner et al. 2016, 2020; Lancelle et al. 2013).

En muchos países del mundo los apicultores producen mieles de un origen floral conocido que, además, son valoradas por presentar alguna característica distintiva como ser el color, sabor o, por ser exclusivamente producidas en una región particular. Se pueden citar algunos ejemplos de mieles con valor agregado, que también son las más caras del mundo; así, en Europa, la miel de acacia [Robinia pseudoacacia L.] es la más clara, suave y de cristalización muy fina, la miel de madroño [Arbutus unedo L.] es una de las pocas mieles amargas, mientras que en Nueva Zelanda la miel de manuka [Leptospermum scoparium J.R. Forst. \& G. Forst.] es valorada por sus extraordinarias propiedades antibacterianas y en Chile la miel de ulmo [Eucryphia cordifolia Cav.] por sus propiedades balsámicas, antibacterianas y fungicidas.

El conocimiento del origen floral y su relación con las propiedades de las mieles es de interés para los apicultores, ya sea por su valor en sí mismo, y porque dicha información es útil como herramienta de comercialización al momento de poner en valor un producto natural como la miel de abejas. En este sentido y desde hace un tiempo, se vienen realizando diversas actividades de divulgación y transferencia, a través de exposiciones y charlas en el marco de Jornadas y Encuentros Apícolas tales como: Jornada Apícola del Impenetrable en J.J. Castelli (Chaco), Semana de la Miel (Corrientes y Chaco) y la Fiesta de la Miel en Saladas (Corrientes). Como así también, mediante la publicación en revistas de divulgación tales como: Campo \& Abejas (Salgado 2005), Espacio Apícola (Salgado 2006b, Salgado y Zago 2008) y Apicultura Sin Fronteras (Salgado 2007). Además, en los últimos años se han fortalecido las prácticas extensionistas mediante la participación en el Programa "La Universidad en el Medio" de la Secretaría General de Extensión Universitaria (UNNE). Los mismos, se enfocaron en la realización de diversas actividades, orientadas a dar respuesta a varios objetivos los cuales apuestan, siempre, al fortalecimiento del sector apícola. 
Salgado Laurenti, C.R. et al.: Acciones de vinculación y transferencia en el sector apícola.

\section{Antecedentes locales}

Los estudios realizados para el conocimiento y caracterización de las mieles de la región representan un recurso útil para diferenciar las mieles y lograr posiciones más competitivas en el mercado, permitiendo agregar valor al producto. Tal es así que, los apicultores acuden a diferentes recursos para distinguirse. En el Chaco, algunas cooperativas y asociaciones han logrado obtener mayor precio de venta diferenciando miel orgánica respecto de la miel convencional.

Es importante destacar que en la zona prevalece el trabajo interinstitucional que involucra profesionales y agentes de INTA, INTI, Ministerio de la Producción, UNNE, entre otras instituciones y Programas, como Cambio Rural y PROSAP, acompañando a los apicultores.

La apicultura practicada en zonas protegidas y con características peculiares, como los sitios Ramsar y localidades cercanas, constituye una alternativa de diferenciación y fuerte aliada en la conservación de la biodiversidad. En este sentido, dos cooperativas (COPAP [Margarita Belén] y Los Palmares [Basail]) y una asociación apícola (Asociación de Productores Apícolas [Charadai]) trabajan en forma conjunta para alcanzar las metas que premitan obtener valor agregado para las mieles que producen. Cabe aclarar que la localidad de Charadai no se encuentra dentro del sitio Ramsar, sin embargo se incluye porque los productores trabajan en forma conjunta y en consecuencia se realizaron las mismas acciones. Las tres agrupaciones son relativamente jóvenes, COPAP se formó en el año 2005, Los Palmares en 2009 y la Asociación de Productores Apícolas en 2010. En el transcurso de estos años, los grupos de productores se han afianzado y han formalizado institucionalmente la organización de las Cooperativas y la Asociación; proceso en el que, desde su creación, fueron apoyados por el INTA con el enfoque de desarrollo territorial.

\section{Breve descripción de la situación abordada}

En el sector productivo. En los últimos años, la zona de producción ha registrado dos problemas recurrentes; el primero son los bajos rendimientos de producción ( $14 \mathrm{~kg}$ miel/colmena/año) y el segundo está relacionado con la calidad de la miel obtenida. El primer problema puede estar relacionado con el desconocimiento de las curvas de floración de la vegetación circundante a los apiarios. Los apicultores podrían realizar una mejor planificación de la producción ajustando las tareas de preparación de las colmenas y un mejor aprovechamiento de los recursos (polen y néctar), en función al conocimiento sistematizado de las curvas de floración. En este sentido, además de saber cuándo, también es importante conocer qué recursos aportan las distintas especies; ya que el polen es importante para el desarrollo de las larvas en la cámara de cría y el néctar para la producción de miel. Por otro lado, el segundo problema puede estar relacionado con la necesidad de reforzar las buenas prácticas de manufactura. La deficiencia en algunos parámetros de calidad, por ejemplo, altos porcentajes de humedad, puede deberse a que se extrae la miel en forma anticipada a la maduración y operculado de los cuadros. La mezcla de miel "verde" o inmadura con miel madura, favorece el incremento de la humedad del producto final. En este punto es importante tener en cuenta que la miel es un producto biológico, por lo que el exceso de humedad genera condiciones que favorecen el desarrollo de microorganismos que deterioran el producto y no es viable para su consumo. Esta situación debe ser controlada y revertida para garantizar la calidad del producto final.

En el sector educativo. La población más permeable a recibir información y adoptar rápidamente nuevos hábitos está constituida por los niños en la primera infancia. Por ello, la planificación de actividades orientadas a dicho sector, para ser llevadas a cabo principalmente por los estudiantes de grado que intervienen en las acciones extensionistas, es uno de los objetivos comunes que persiguen los proyectos de extensión. En este marco, los estudiantes colaboran en la planificación de charlas y juegos didácticos para trabajar con los niños en las escuelas de la zona o en otros espacios pertinentes, con el objetivo de fomentar el consumo de miel e informar acerca de los beneficios de ello. Además, se busca constantemente transmitir sobre el valor de las abejas como polinizadores y la importancia de su protección a favor de la conservación de la biodiversidad.

En el sector social. En el marco de eventos socio-culturales el grupo extensionista participa activamente con la presentación de un stand mediante el cual es posible llegar a un público más numeroso y amplio, despertando la curiosidad de conocer el mundo de las abejas y alentando al consumo de sus productos. Las actividades abordadas se centran en charlas, degustaciones de mieles, difusión, juegos, etc. 
El trabajo extensionista llevado a cabo en los últimos años se focalizó en cumplir los siguientes objetivos generales: -Fortalecer la producción apícola, mediante el conocimiento de los recursos vegetales, para incrementar los volúmenes de producción garantizando la calidad fisicoquímica y microbiológica de la miel. Brindar a los productores estrategias necesarias para generar valor agregado a la miel que se obtiene en la zona. -Fortalecer el manejo de los apiarios en función al aprovechamiento de la curva de floración, aumentando el volumen de producción de miel y garantizar parámetros de calidad dependientes de las buenas prácticas. -Poner en valor la miel producida en el Sitio Ramsar (Humedales Chaco), valioso por su alta diversidad específica. Incorporar hábitos saludables mediante el estímulo al consumo de miel desde la temprana edad.

Para cumplir con estas metas planteadas, como también abordar la problemática del sector apícola y presentar estrategias a fin de contribuir a revertir la situación, mediante el trabajo integrado de varias instituciones, se presentaron tres (3) Proyectos de Extensión, anuales y consecutivos, en el marco del Programa "La Universidad en el Medio" de la Secretaría General de Extensión Universitaria - UNNE convocatorias 2016 (ejecución enero a diciembre 2017), 2017 (ejecución abril 2018-2019) y 2018 (ejecución abril 2019-2020).

\section{METODOLOGÍA Y RESULTADOS OBTENIDOS}

Las acciones extensionistas interdisciplinarias e interinstitucionales mencionadas en el presente trabajo fueron llevadas a cabo a partir de una serie de recursos, los cuales se detallan a continuación.

\section{Insumos y fuentes de financiamiento}

Fue necesario contar con información obtenida de producciones previas (conocimientos y resultados publicados en revistas científicas y de divulgación). Recursos materiales, facilitadores TIC, infraestructura, capital humano y conocimientos previos de los apicultores. El financiamiento provino de los Proyectos presentados y adjudicados del Programa "La Universidad en el Medio": Proyectos 0149-2016 (2017-2018), 290-2018 (20182019) y 194-2019 (2019-2020).

\section{Características de la población abordada}

Los destinatarios directos del proyecto son productores apícolas, consolidados en seis grupos del Programa Cambio Rural (INTA Colonia Benítez) y dos Cooperativas: "COPAP" ubicada en la localidad de Margarita Belén y "Los Palmares" en Basail y la "Asociación de Productores Apícolas" de Charadai. La actividad apícola es principal y está asociada a otras actividades de producción primaria para auto sustento y venta en el mercado local. Por lo que sus ingresos económicos dependen principalmente de estas actividades. Cabe destacar que los productores cuentan con el apoyo y asesoramiento técnico del Programa Cambio Rural del INTA. Por otra parte, pero no menos importante, las actividades extensionistas están dirigidas a público general y al sector académico (de diferentes niveles educativos: inicial, primario y universitario).

\section{Organización de las actividades}

En el marco del desarrollo de las acciones extensionistas se llevaron a cabo actividades en torno a los siguientes ejes:

Difusión y Jornadas: se realizaron charlas al sector educativo-académico y público general, Talleres formativos con productores y se participó en Jornadas de Extensión y Científicas. Además, se desarrollaron Disertaciones en distintos ámbitos, a cargo de los docentes del equipo de trabajo. Se participó en distintas ediciones de la Semana de la Miel, Expo Miel y el Patio de la Miel, mediante la presentación de un stand informativo para público general, acompañado de juegos didácticos para niños, degustaciones de mieles, charlas, etc. (Figs. 1 y 2 ).

Capacitación: se dictaron Pasantías de Investigación y Cursos de Posgrado y de Actualización, con referencia al análisis de calidad de mieles. Por otro lado, se logró la articulación del equipo de los Proyectos con otras instituciones en el Taller Regional NEA para la elaboración de propuestas en el marco del Plan Estratégico Apícola - PEA 2030; como así también se asistió a Talleres referentes a las actividades extensionistas en el marco de las Jornadas de Extensión Universitaria (Fig. 3).

Formación de RRHH: se alentó a la participación y consolidación del trabajo grupal de los estudiantes de grado de las unidades académicas involucradas en los Proyectos (Fig. 3). 

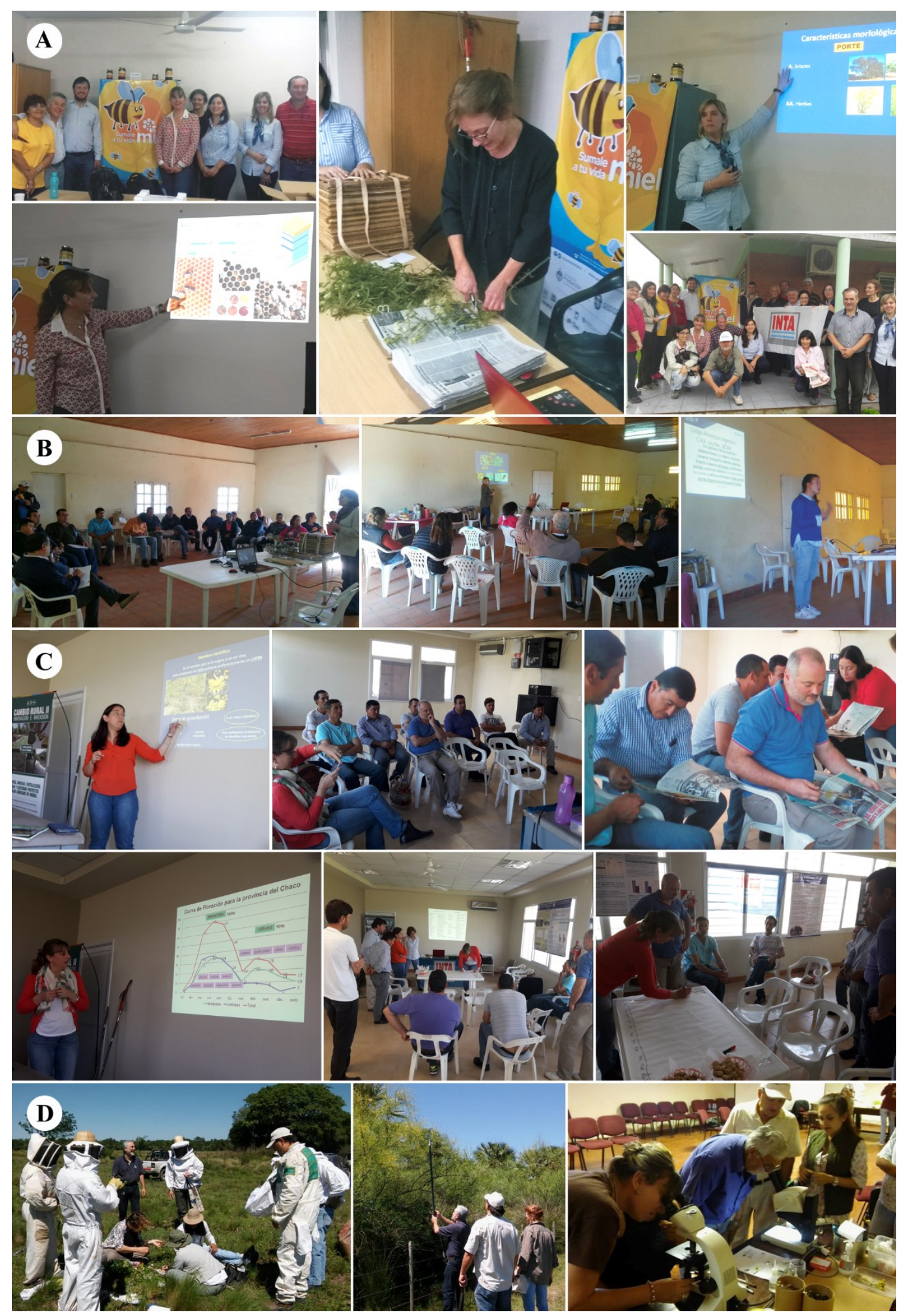

Figura 1. Talleres formativos: Flora Melífera. A. COPAP, Margarita Belén. B. Asociación de Productores Apícolas de Charadai. C. Cooperativa "Los Palmares", Basail. D. INTA Colonia Benítez. 

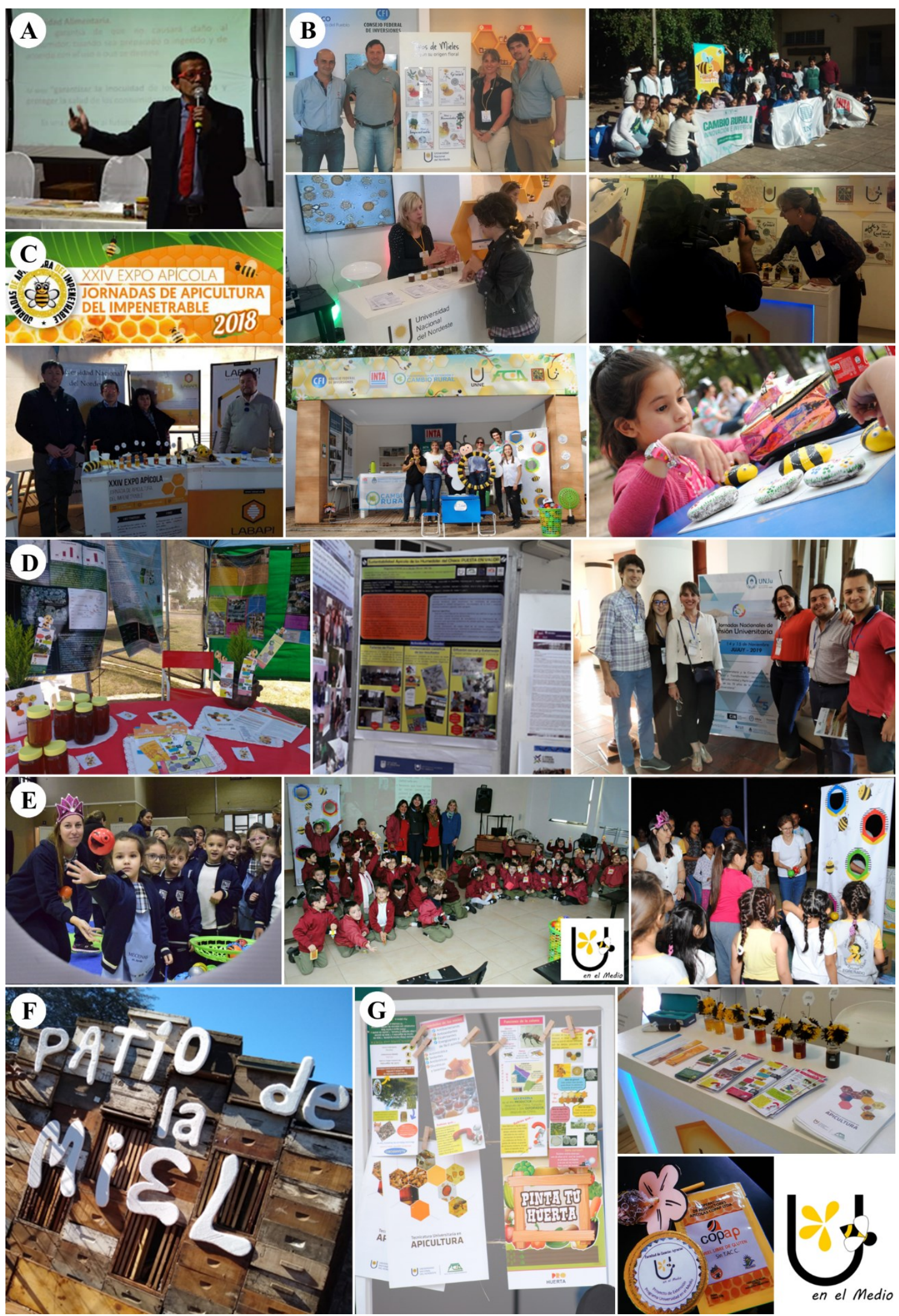

Figura 2. Difusión y Jornadas. A. Disertación UCP: Agregando Valor: Calidad e Inocuidad. B. Semana de la Miel; Eds. 2017, 2018, 2019 (Stand informativo y juegos). C. Jornada Expo Apícola. D. Jornadas de Extensión Universitaria (Regionales y Nacionales); Eds. 2017, 2018, 2019. E. Charlas sector educativo (nivel inicial y primario). F. Participación evento, Miraflores, Chaco. G. Folletería, suvenires y logo. 


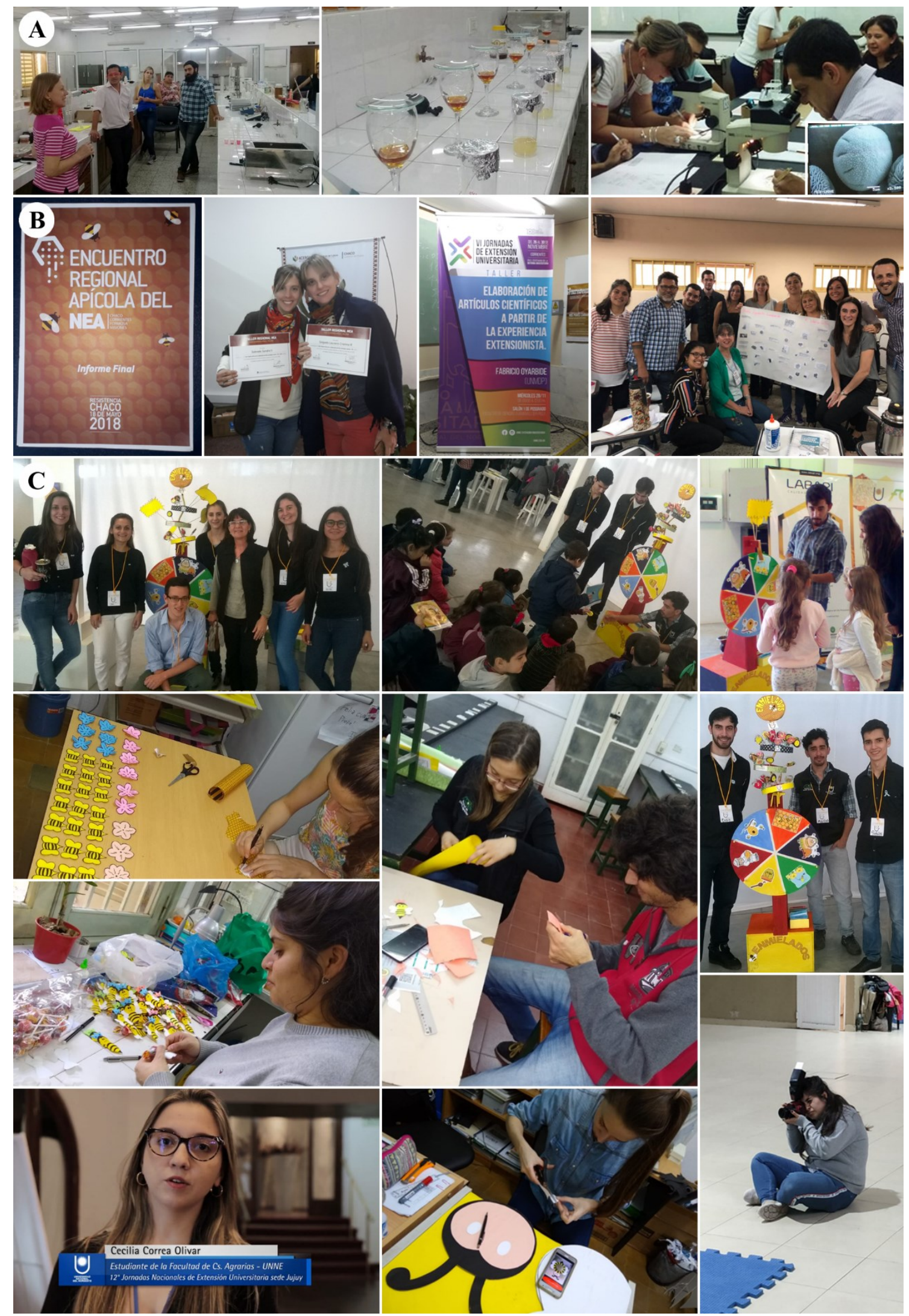

Figura 3. Capacitación y Formación del Equipo Extensionista (Docentes y Estudiantes). A. Cur so de Posgrado: "Calidad de la Miel: aspecto sensorial, fisicoquímico y polínico" (FaCENA - FCA). B. Articulación entre agentes de los Proyectos y otras instituciones (Taller Regional NEA - PEA 2030); Talleres de actividades extensionistas en el marco de las Jornadas Universitarias. C. Participación de los estudiantes en distintas actividades y eventos. 


\section{Acciones emprendidas}

Se organizaron en distintos grupos de tareas, las que se detallan a continuación:

Organización y ejecución de Encuentros y Talleres con apicultores. Docentes de la Universidad y Técnicos de diferentes Unidades Ejecutoras, realizaron Talleres de trabajo con productores con el objetivo de ajustar la curva de floración de las especies melíferas más importantes, identificadas en los apiarios. En conjunto con Técnicos del INTA, se realizó una programación productiva, integrando el conocimiento de la oferta de polen y néctar y el desarrollo de las colonias de abejas. En estos encuentros, llevados a cabo en las tres localidades seleccionadas, se realizaron exposiciones orales mediante recursos informáticos y audiovisuales sobre especies melíferas, los recursos que éstas ofrecen (polen y/o néctar) y las curvas de floración de las mismas. Se transmitió información sobre el origen floral de las mieles chaqueñas comprobado con análisis palinológicos y sus características sensoriales. Se informó además acerca de las buenas prácticas de manufactura y destacó la importancia de su aplicación para garantizar la calidad del producto alimenticio.

Por su parte, Técnicos del INTA e INTI desarrollaron actividades en el Programa Cambio Rural asistiendo a los productores, colaboraron en el trabajo de campo y en todas las actividades que involucraron logística para el llevar a cabo los Talleres programados (invitación a productores, traslado de docentes y estudiantes a los lugares de reunión y a los apiarios, etc.). En el marco de estas actividades, abordaron especialmente las cuestiones referidas al manejo de apiarios y articularon tareas de manera integrada con Docentes, Técnicos y Estudiantes de la UNNE, para fortalecer el desarrollo de la actividad apícola de la zona.

Tareas de Laboratorio. El trabajo de análisis de muestras de miel, para corroborar el origen floral y evaluar los parámetros fisicoquímicos y microbiológicos, se realizó en los laboratorios de Palinología de FCA y LABAPI de FaCENA respectivamente. Los resultados obtenidos fueron socializados con los productores durante los talleres, en los que se realizó la devolución de los mismos en forma oral e impresa.

Entrenamiento de los estudiantes extensionistas. Los estudiantes participantes fueron previamente instruidos para colaborar en todas las actividades planificadas en los proyectos. Realizaron diversas tareas, entre las que se pueden mencionar el diseño y la producción de material gráfico, folletos y audiovisuales para difusión de información. Además, durante la ejecución de los encuentros y talleres desarrollaron actividades de asistencia en general y coordinación, instancias en las que tuvieron oportunidad de explicar contenidos y ser de apoyo a los grupos de productores. Los estudiantes están enfocados a todo tipo de actividades operativas y se los considera como agentes facilitadores en las charlas con alumnos de escuelas primarias y secundarias.

\section{Tareas llevadas a cabo}

Para alcanzar los objetivos generales se plantearon objetivos particulares, los cuales se lograron mediante el desarrollo de distintas actividades y tareas. Estas fueron diseñadas, programadas y ejecutadas por distintos actores extensionistas y en distintos momentos durante los tres años de ejecución de los Proyectos de "La Universidad en el Medio". Algunos objetivos y su correspondiente tarea cumplida se resumen en la Tabla 1.

\section{Capital humano}

El equipo de trabajo es interdisciplinario e interinstitucional y está conformado por Docentes, Técnicos y Estudiantes en un 44\% LABAPI y FaCENA-UNNE, 28\% FCA-UNNE e Instituto Agrotécnico P.F. Godo, 22\% INTA Colonia Benítez, 3\% INTI y 3\% IBONE-CONICET. La información detallada de todos los participantes del equipo extensionista involucrado en los Proyectos se detalla en la Tabla 2.

\section{Resultados obtenidos}

Las acciones de intervención del equipo de trabajo interdisciplinario e interinstitucional alcanzaron a unos 50 apicultores, a quienes se logró sensibilizar respecto de la importancia de conocer las especies de plantas melíferas de su zona. Como así también poner en valor los recursos que obtienen las abejas a partir de ellas y el destino dentro de la colonia: polen y néctar. Sin dudas, esto contribuye a fortalecer el manejo de los apiarios en función del aprovechamiento de la curva de floración a fin de aumentar el volumen de producción de miel y garantizar los parámetros de calidad que dependen de las buenas prácticas. 
Salgado Laurenti, C.R. et al.: Acciones de vinculación y transferencia en el sector apícola.

Tabla 1. Relación entre los objetivos particulares cumplidos y las actividades realizadas.

\section{Objetivos específicos}

Tareas/Actividades

\begin{abstract}
Valorizar la diversidad de las especies botánicas que definen el tipo de miel de la zona, conocer los recursos que ofrece cada una (polen y/o néctar) y ajustar las curvas de floración.
\end{abstract}

Facilitar información a los productores mediante artículos de divulgación breves e ilustrados.

Transmitir buenas prácticas de manufactura y la impor-

Comunicar sobre parámetros de calidad de la miel. tancia de su aplicación.

Taller de Flora Melífera y métodos de colección y herborización de plantas (COPAP, Margarita Belén, 2017; Asociación de Productores Apícolas, Charadai, 2017; Coop. "Los Palmares", Basail, 2017; INTA Colonia Benítez, 2018; Santa Sylvina, 2019).

Confección e impresión de texto informativo, breve e ilustrado en folletos, afiches, señaladores, entre otros.

Charla informativa a cargo de Técnicos del INTA e INTI.

Disertación “Agregando Valor: Calidad e Inocuidad”. Realizado en la Universidad de la Cuenca del Plata, Corrientes. A cargo del Esp. (Lic.) Daniel Ruiz Díaz.

Desarrollo de Cursos de Posgrado:

"Calidad de Miel: aspecto sensorial, fisicoquímico y polínico" (FaCENA - FCA). 2017.

Analizar los parámetros fisicoquímicos y microbiológicos en muestras de miel.
"Buenas Prácticas en el Análisis de Parámetros fisicoquímicos de Mieles de Abejas" (FaCENA - FCA). 2018.

Desarrollo de Pasantía de Investigación no rentada:

"Entrenamiento en el análisis físicoquímico y sensorial en mieles de abeja” (LABAPI - FaCENA). 2019.

Visita al LABAPI

Transmitir las propiedades nutricionales de la miel.

Difundir información del origen floral de las mieles chaqueñas y sus características sensoriales.

Estimular el consumo interno de miel y subproductos mediante acciones de difusión.
Participación:

Semana de la Miel (Chaco y Corrientes). Ediciones: 2017, 2018 y 2019. Stand informativo.

Jornadas de Extensión de la UNNE. Ediciones: 2017, 2018 y 2019. Presentación modalidad panel y oral.

$12^{\circ}$ Jornadas Nacionales de Extensión Universitaria (Jujuy, 2019). Presentación modalidad panel y oral.

Tecnópolis Federal. Edición 2017. Stand informativo.

Jornada de difusión en la Semana del Ambiente - Edición 2018: Apicultura saludable. Corrientes. A cargo de la Dra. Cristina R. Salgado.

XXIV Expo Apícola - Jornada de Apicultura del Impenetrable. Edición 2018. J.J. Castelli, Chaco. Stand informativo, presentación paneles.

XII Congreso Latinoamericano de Apicultura. Montevideo, Uruguay. 2018. Presentación modalidad panel.

Eventos: Expo Miel. Santa Sylvina, Chaco. 2018. El Patio de la Miel. Miraflores, Chaco. 2019. Stand informativo.

Diseño del Logo identificador del Grupo y Proyectos.

Formar grupos de trabajo para diseñar folletería y distintos materiales de difusión.

Desarrollar actividades creativas orientadas a los niños para difundir la actividad apícola y el consumo de miel.

Fomentar en los jóvenes estudiantes el compromiso social para su formación integral como futuros profesionales.

Diseño y armado de juego didáctico para niños a cargo de los estudiantes. Entrega de suvenires a todos los participantes.

Participación activa en la planificación, organización y ejecución de todas las actividades de los Proyectos.

Docentes: Articulación del equipo del Proyecto con otras instituciones en el Taller Regional NEA para la elaboración de propuestas en el marco del Plan Estratégico Apícola - PEA 2030. Resistencia, Chaco. 2018. 
Tabla 2. Listado del capital humano involucrado en los Proyectos de Extensión, pertenencia e instituciones intervinientes.

\begin{tabular}{|c|c|c|}
\hline Integrantes & Formación académica/Actividad & Institución de pertenencia \\
\hline Aguirre Rollet, Marcos & Ingeniero Agrónomo & FCA - UNNE \\
\hline Badán, Nancy M. & Téc. Univ. en Calidad de Prod. Apícolas & LABAPI - FaCENA - UNNE \\
\hline Barboza, Enzo & Estudiante de Ing. Agronómica & FCA - UNNE \\
\hline Behmetiuk, Maximiliano & Estudiante de Ing. Agronómica & FCA - UNNE \\
\hline Benítez Núñez, Briant S. & Estudiante de Ing. Agronómica & FCA - UNNE \\
\hline Berli, Ayelen J. & Estudiante Lic. en Cs. Químicas & FaCENA - UNNE \\
\hline Bertona, Vannina & Bioquímica & FaCENA - UNNE \\
\hline Boscarino, Sebastián & Estudiante de Ing. Agronómica & FCA - UNNE \\
\hline Brenn, Jazmin & Estudiante Lic. en Cs. Químicas & FaCENA - UNNE \\
\hline Changazzo, Juliana A. & Ingeniera Agrónoma & FCA - UNNE \\
\hline Churruarín, Matías & Estudiante de Ing. Agronómica & FCA - UNNE \\
\hline Codutti, Daniel & Estudiante Tec. Univ. Apicultura (FCA-UNNE) & Cambio Rural - INTA \\
\hline Correa Olivar, Laura C. & Estudiante Ing. Agronómica & FCA - UNNE \\
\hline Cuevas, Isabel & Promotora Asesora & Cambio Rural - INTA \\
\hline Fechner, Diana & Lic. en Cs. Químicas & FaCENA - UNNE \\
\hline Franchescutti, Flavia & Ingeniera Agrónoma & INTA Colonia Benítez \\
\hline Gaiad, Emilio & Ingeniero Agrónomo & FCA - UNNE \\
\hline Gauto, Silvana Y. & Estudiante Lic. en Cs. Biológicas & FaCENA - UNNE \\
\hline Geijo, Rubén & Lic. en Administración Rural & Cambio Rural - INTA \\
\hline Gimenez, Javier & Estudiante Tec. Univ. Apicultura (FCA-UNNE) & Cambio Rural - INTA \\
\hline González, Ma. Florencia & Estudiante de Bioquímica & FaCENA - UNNE \\
\hline Huber, Yamila D. & Estudiante de Bioquímica & FaCENA - UNNE \\
\hline Insaurralde, Oscar & Téc. Univ. en Calidad de Prod. Apícolas & LABAPI - FaCENA - UNNE \\
\hline Luna, Francisco & Estudiante de Ing. Agronómica & FCA - UNNE \\
\hline Lutz, Cinthia C. & Estudiante Lic. en Cs. Químicas & FaCENA - UNNE \\
\hline Mazepa, Cristian I. & Estudiante de Ing. Agronómica & FCA - UNNE \\
\hline Melgrati, Rene & Productor apícola & Cambio Rural - INTA \\
\hline Miguel, Laila M. & $\begin{array}{l}\text { Dra. de la UNNE, Especialidad Biología. Docente } \\
\text { FaCENA }\end{array}$ & FaCENA - UNNE \\
\hline Paredes, Jonhatan A. & Prof. en Cs. Química y del Ambiente & FaCENA - UNNE \\
\hline Pieszko, Gelina & Prof. en Biología. Técnica CPA IBONE & IBONE - UNNE/CONICET \\
\hline Ruiz Díaz, Juan D. & Esp. en Calidad. Docente FaCENA & LABAPI - FaCENA - UNNE \\
\hline Rusas, Víctor & Técnico Universitario Apícola & Cambio Rural - INTA \\
\hline Sáez, Roberto & Contador & INTA Colonia Benítez \\
\hline Salgado, Cristina R. & Dra. de la UNNE en Recursos Naturales. Docente FCA & FCA - UNNE \\
\hline Scherf, Romina & Extensionista & INTI \\
\hline Sirio, Andrea & Bioquímica & Inst. Agrot. Fuentes Godo \\
\hline Sobrado, Sandra V. & $\begin{array}{l}\text { Dra. de la UNNE, Especialidad Biología. Docente } \\
\text { FaCENA Inv. Asistente CONICET }\end{array}$ & $\begin{array}{l}\text { FaCENA - UNNE. IBONE - } \\
\text { UNNE/CONICET. }\end{array}$ \\
\hline Vagabculov, Javier & Lic. en Administración Rural & INTA Colonia Benítez \\
\hline Vázquez, Francisco A. & Dr. en Química & FaCENA - UNNE \\
\hline
\end{tabular}


Por otra parte, se incluyeron acciones extensionistas destinadas al público en general, con la finalidad de fomentar el consumo de miel de abejas y poner en valor sus propiedades nutricionales. En este sentido, se espera disminuir el consumo de azúcar blanca reemplazándola por el consumo de miel especialmente en los niños en edad escolar.

Respecto a los estudiantes de grado involucrados, se logró despertar en ellos la vocación extensionista y el afianzamiento de diversas habilidades que complementan su formación como futuros profesionales. Cabe destacar que en este sentido, es importante comprendan y valoren la dinámica de trabajo colectivo, búsqueda de información, desarrollo de capacidad creativa e interacción con distintos actores de la comunidad socioproductiva (docentes, alumnos, técnicos, productores, etc.).

Finalmente, la experiencia llevada a cabo y las tareas del grupo extensionista fue dada a conocer mediante la participación en Congresos, Jornadas Extensionistas y Científicas de la UNNE e instancias nacionales.

\section{CONSIDERACIONES FINALES}

Las acciones de intervención realizadas con anterioridad en forma general y masiva constituyen la base para realizar otras más focalizadas. Teniendo en cuenta que los objetivos de la práctica extensionista buscan esencialmente un cambio de conducta, es esperable que el impacto de los resultados se visualice a largo plazo y en pulsos lentos de desarrollo, hasta poder identificar cambios significativos. Sin embargo, las actividades extensionistas, intensificadas en los últimos tres años en una población reducida y determinada, han manifestado algunos resultados que se detallan a continuación. En el:

\section{Sector productivo:}

- Forjamos interés en los productores apícolas por el conocimiento de la flora melífera de su zona de producción, generando información que les sirva para diferenciar los tipos de mieles que se producen en las 3 localidades de la zona a fin de obtener un valor agregado.

- Sensibilización respecto al valor de conocimientos teórico-prácticos durante los Talleres formativos a apicultores de Margarita Belén, Basail y Charadai.

\section{Ámbito académico:}

- Fortalecimiento de lazos mediante trabajo en equipo entre los diferentes actores y las Instituciones intervinientes.

- Desarrollo de actitudes de responsabilidad en los estudiantes de las Facultades de Ciencias Agrarias y Ciencias Exactas y Naturales y Agrimensura, en el trabajo colaborativo y el compromiso social preparándolos para su desempeño profesional.

- Participación en las Jornadas de Extensión Universitaria de la UNNE y en otras jornadas nacionales.

- Desarrollo de un Curso de Calidad de Mieles en forma conjunta con las 2 Unidades Académicas.

\section{Sector Público:}

- Participación en las Jornadas de la Semana de la Miel "Sumale Miel a tu Vida" y en Tecnópolis Federal. Eventos en los que se llegó a un público más numeroso y amplio, fomentando el consumo de miel y despertando la curiosidad por el mundo de las abejas. En tales instancias se interactuó con todas las edades, a través de: juegos didácticos, folletería, análisis de muestras de mieles mediante microscopía óptica, degustación de distintos tipos de mieles, entre otras.

\section{Proyección de la actividad extensionista}

El equipo de trabajo se renueva periódicamente, incorporando nuevos integrantes y proyecta tener continuidad respondiendo a las demandas del sector productivo interesado. En el futuro, será necesario completar actividades que se planificaron y no pudieron ser cumplidas en su totalidad, como también planificar la ejecución de otras nuevas. 


\section{BIBLIOGRAFÍA}

Bertona, V., Fechner, D.C., Moresi, A.L., Pellerano, R.G., Ruiz Díaz, J.D. y Vazquez, F.A. (2014). Calidad Físicoquímica de mieles recolectadas en la Provincia de Corrientes, ALDEQ (Anuario Latinoamericano de Educación Química). Año XXVII- Nº XXIX: 95-99. 2013-2014.

Fechner, D.C., Moresi, A.L., Ruiz Díaz, J.D., Pellerano, R.G. y Vazquez, F.A. (2016). Multivariate classification of honeys from Corrientes (Argentina) according to geographical origin based on physicochemical properties. Food Bioscience 15: 49-54.

Fechner, D.C., Hidalgo, M.J., Ruiz Díaz, J.D., Gil, R.A. y Pellerano, R.G. (2020). Geographical origin authentication of honey produced in Argentina. Food Biosciencie 33 (100483). https://doi.org/10.1016/ j.fbio.2019.100483.

González González, G. y Fernández- Larrea, M. (2019). La responsabilidad social de la universidad: antecedentes, conceptos, tendencias y retos de la extensión universitaria. Revista Compromiso Social 1 (1): 11-22.

Lancelle, H.G., Fechner, D.C., Moresi, A.L., Armua, O.M., Badan, N.M. y Vazquez, F.A. (2013). Physicochemical characteristics and quality analysis of commercial honeys of Apis mellifera produced by artisanal beekeepers in Corrientes (Argentina). Trade Science Inc. Natural Products: An Indian Journal 9(6): 228-236.

Proyecto Resol. 195/17. Convocatoria 2016. Ejecución 01/01/2017-31/12/2017. Universidad en el Medio. Fortalecimiento del Sector Apícola del Sudeste Chaqueño. Facultad de Ciencias Agrarias. Director: Cristina Salgado. Co-Director: Daniel Ruiz Díaz. Coordinador: Victor Rusas.

Proyecto Resol. 290/18. Convocatoria 2017. Ejecución 25/04/2018-25/04/2019. Universidad en el Medio. Sustentabilidad Apícola de los Humedales del Chaco: puesta en VALOR. Facultad de Ciencias Agrarias. Director: Cristina Salgado. Co-Director: Daniel Ruiz Díaz. Coordinador: Victor Rusas.

Proyecto Resol. 194/19. Convocatoria 2018. Ejecución 01/05/2019-01/05/2020. Universidad en el Medio. Mieles de Humedales: potencial florístico y calidad. Facultad de Ciencias Agrarias. Director: Cristina Salgado. Co-Director: Sandra V. Sobrado. Coordinador: Victor Rusas.

Salgado, C.R. y Maidana, J.F. (2014). Physicochemical characterisation of honey produced in the Chaco Province (Argentina). Rev. FCA UNCUYO 46(2): 191-201.

Salgado, C.R. (2005). Características botánicas de las mieles del Chaco. Campo \& Abejas. 43: 7-8.

Salgado, C.R. (2006a). Flora melífera de la provincia de Chaco. Editado por PROSAP y Ministerio de Producción del Chaco: 60 págs.

Salgado, C.R. (2006b). Flor a melífer a del Chaco. Espacio Apícola 72: 26-31.

Salgado, C.R. (2007). Las mieles del Chaco: caracterización botánica. Apicultura Sin Fronteras. Edición Electrónica. Año II N¹2. Página 10.

Salgado, C.R. y Zago, L. (2008). Mieles del Talanaqonat. Espacio Apícola 83: 12-20.

Salgado, C.R., Pieszko, G.E. y Tellería, M.C. (2014). Apor te de la melisopalinología al conocimiento de la flora melífera de un sector de la Provincia Fitogeográfica Chaqueña, Argentina. Boletín de la Sociedad Argentina de Botánica 49(4): 513-524.

Salgado, C.R. y Pire, S.M. (1998). Análisis polínico de mieles del Noroeste de la provincia de Corrientes (Argentina). Darwiniana 36(1-4): 87-93.

Salgado, C.R. y Pire, S.M. (1999). Contribución al conocimiento del contenido polínico de mieles de Corrientes (Argentina). Ameghiniana, A.P.A. publicación especial 6: 95-99.

Salgado, C.R., Tellería, M.C. y Coronel, J.M. (2017). Botanical and geografphical origin of honey from the dry and humid Chaco ecoregions (Argentina). Grana http:// dx.doi.org/10.1080/00173134.2016.1276619 